\title{
Perspectivas en la ciencia y tecnología de cementos
}

\section{Perspectives in the Science of Technology of Cements}

\author{
F. P. GLASSER \\ Department of Chemistry, University of Aberdeen \\ Old Aberdeen, AB9 2UE, Scotland
}

\section{RESUMEN}

Se han realizado avances muy rápidos en la ciencia y tecnología de la fabricación y el uso del cemento. La duración prevista de esta conferencia permite sólo destacar unos pocos temas seleccionados. El mayor estímulo para la investigación en el campo del cemento es debida a factores económicos tanto en la fabricación como en el uso; to que sugiere ciertas líneas de investigación tanto a nivel fundamental como aplicada.

Las áreas que serán discutidas aquí, relacionan cinco temas que son resumidos en la tabla 1.

\section{SUMMARY}

Rapid advances are being made in the science and technology of cement manufacture and use are being made. Time and space permit me to highlight only a few selected topics. However, the main stimulus for cement research arises from economic factors both in

manufacture and use which in turn suggest certain lines of research, both fundamental and applied. The topical areas which will be discussed here relate to five themes which are summarized in Table 1.

\section{ECONOMIAS EN LA PRODUCCION Y USO DEL CEMENTO}

Los avances conseguidos en la transferencia de calor en el horno rotatorio, así como la introducción del proceso de fabricación por via seca y el uso de precalentadores con suspensión, han permitido bajar sustancialmente el consumo de energía necesario para la clinkerización. Los cementos Portland no son materiales excesivamente energéticos respecto a otros como el acero o el aluminio, pero no obstante requieren una gran cantidad de energía obtenida a través de fuel y electricidad; disminuir esa cantidad de energía sería muy deseable para mantener o mejorar la posición de competitividad de los cementos.

Es poco probable, por motivos termodinámicos que los ahorros de energía en la cocción puedan ser mejorados mucho, con respecto a las condiciones más adecuadas que actualmente se utilizan. Examinaré algunas iniciativas.

"Cementos de baja energía" pueden ser

\section{ECONOMICS OF CEMENT MAKING AND USE}

Advances in heat transfer in rotary kilns, the widespread introduction of dry process kiln and the general use of suspension preheaters have substantially lowered the energy requirements for clinkering. Portland cements are not an energy rich material relative to steel or aluminium, but they nevertheless represent a large specific energy input of fossil fuels and electricity and a further decrease in specific energy requirements would be desirable to maintain or improve the competitive position of cements. It is unlikekey on thermodynamic grounds that the economics of kiln operation can be much improved, relative to the best present practices. Therefore progress in this respect must be made in other directions. l explore some initiatives. "Low energy cements" may be defined as those which, when compared with OPC on the basis of equal performance, require less energy input. Several aproaches to low-energy cementitious materials are outlined in Table 2. We may comment briefly on these. 
definidos como aquellos que comparados con los OPC $\left(^{\star}\right)$ y a igualdad de prestaciones, requieren menor energía. Algunas aproximaciones a materiales conglomerantes de baja energía están descritos en la tabla 2 . Nosotros los comentaremos brevemente.

En el proceso normal de fabricación del cemento, $\mathrm{Al}_{2} \mathrm{O}_{3}$ y $\mathrm{Fe}_{2} \mathrm{O}_{3}$ actúan como fundentes. Su presencia disminuye la temperatura de aparición de líquido, respecto a una mezcla de $\mathrm{CaO}$ y $\mathrm{SiO}_{2}$. Los principios en los que se basa esto se pueden conocer por el estudio del sistema

$\mathrm{CaO}-\mathrm{Al}_{2} \mathrm{O}_{3}-\mathrm{Fe}_{2} \mathrm{O}_{3}-\mathrm{SiO}_{2}$. Mezclas de $\mathrm{Fe}_{2} \mathrm{O}_{3}$ y $\mathrm{Al}_{2} \mathrm{O}_{3}$ son más efectivas que cualquiera de los dos individualmente. Las relaciones entre el contenido, en tanto por ciento en peso del clínker, a temperaturas de clinkerización y la cantidad de líquido formado ha sido representado gráficamente (1). Ya que el líquido en equilibrio con $\mathrm{C}_{3} \mathrm{~S}$ y $\mathrm{C}_{2} \mathrm{~S}$ a una temperatura dada tiene una composición fija que es independiente de la relación $\mathrm{Al}_{2} \mathrm{O}_{3} / \mathrm{Fe}_{2} \mathrm{O}_{3}$, las propiedades de transporte del líquido son constantes e independiente de la relación $\mathrm{Al}_{2} \mathrm{O}_{3} / \mathrm{Fe}_{3} \mathrm{O}_{3}$. Por ello, las fases convencionales del clínker ofrecen relativamente poca diversidad.

(*) Ordinary Portland Cement.

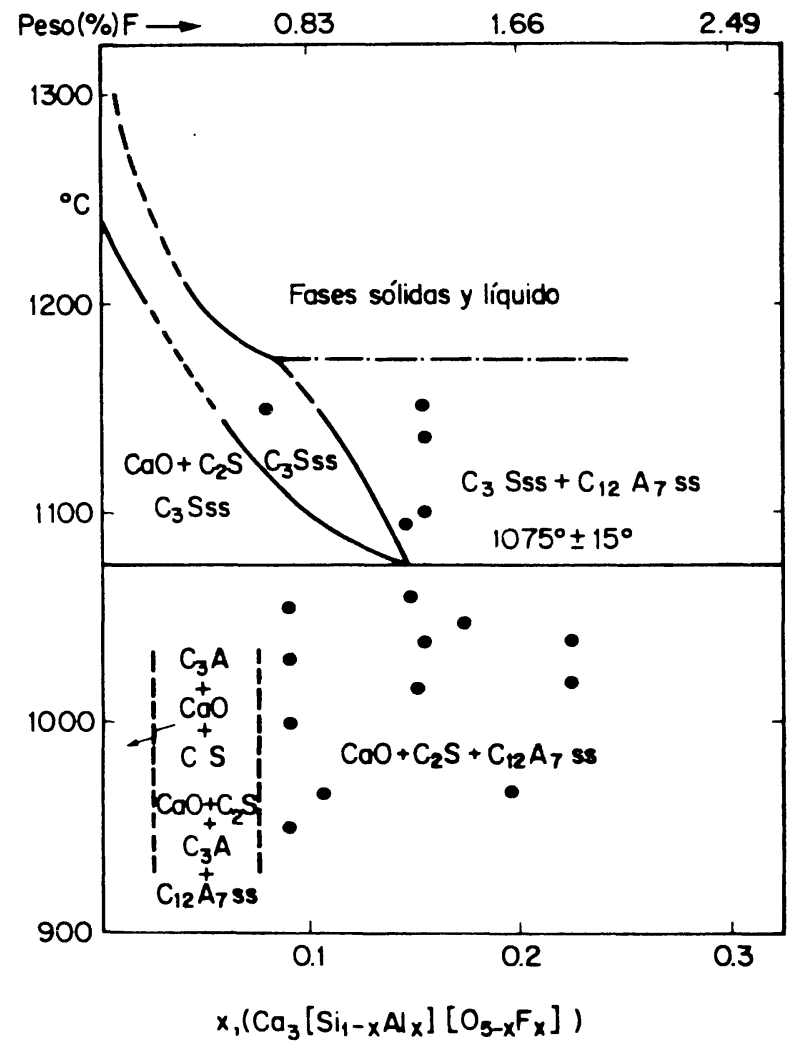

44
In the normal cement burning process, $\mathrm{Al}_{2} \mathrm{O}_{3}$ and $\mathrm{Fe}_{2} \mathrm{O}_{3}$ act as fluxes. Their presence lowers the temperature at which melt formation occurs ive to mixtures of $\mathrm{CaO}$ and $\mathrm{SiO}_{2}$. The principles by which this occurs are well understood as a result of phase studies of the $\mathrm{CaO}-\mathrm{Al}_{2} \mathrm{O}_{3}-\mathrm{Fe}_{2} \mathrm{O}_{3}-\mathrm{SiO}_{2}$ system. Mixtures of $\mathrm{Fe}_{2} \mathrm{O}_{3}$ and $\mathrm{Al}_{2} \mathrm{O}_{3}$ are more effective than either of the individual fluxing agents. The relations between weight $\%$ content of the clinker batch clinkering temperature and amount of liquid formed have been presented in graphical form (1). Since the liquid in equilibrium with both $\mathrm{C}_{3} \mathrm{~S}$ and $\mathrm{C}_{2} \mathrm{~S}$ at a given temperature has a fixed composition which is independent of $\mathrm{Al}_{2} \mathrm{O}_{3} / \mathrm{Fe}_{2} \mathrm{O}_{3}$ ratio, the transport properties of the liquid are constant and also independent of the $\mathrm{Al}_{2} \mathrm{O}_{3} / \mathrm{Fe}_{2} \mathrm{O}_{3}$ ratio. Thus, conventional clinker mineralogies offer relatively limited scope for development.
Fig. 1.-Diagrama seudobinario del sistema $\mathrm{CaO}-\mathrm{Al}_{2} \mathrm{O}_{3}-\mathrm{CaF}_{2}$ mostrando la estabilidad de disoluciones sólidas $\mathrm{Ca}_{3} \mathrm{SiO}_{5}$ a $1.075^{\circ} \mathrm{C}$ por sustituciones $(\mathrm{Al}+\mathrm{F})$ por $(\mathrm{Si}+\mathrm{O})$.

Fig. 1.-Pseudo-binary join in the system $\mathrm{CaO}-\mathrm{Al}_{2} \mathrm{O}_{3}-\mathrm{SiO}_{2}-\mathrm{CaF}_{2}$ showing the stabilization of $\mathrm{Ca}_{3} \mathrm{SiO}_{5}$ solid solutions to $1.075^{\circ} \mathrm{C}$ by combined $(A)+F)$ substitution for $(\mathrm{Si}+\mathrm{O})$ 


\section{POSIBILIDAD DE DESARROLLO DE CEMENTOS DE BAJA ENERGIA}

Un camino para eliminar las condiciones restrictivas es cambiar drásticamente la composición del clínker, para que a las mayores temperaturas de clínkerización, el $\mathrm{C}_{3} \mathrm{~S}$ y $\mathrm{C}_{2} \mathrm{~S}$ no estén, en gran proporción, en equilibrio con la fase líquida. Esto se puede conseguir modificando las condiciones de invarianza isotermal impuesta para composiciones convencionales, por ejemplo, algunos cementos Ferrari que no tienen $\mathrm{C}_{2} \mathrm{~S}$ podrían tener esta condición. La tabla 3 muestra una composición, que después de templada, contiene mayoritariamente $\mathrm{C}_{3} \mathrm{~S}$, $\mathrm{C}_{4} \mathrm{AF}$ y $\mathrm{C}_{3} \mathrm{~A}$. A temperaturas de clinkerización $1.350^{\circ} \mathrm{C}$, el $\mathrm{C}_{3} \mathrm{~S}$ es probablemente la única fase cristalina que coexiste con el líquido. Naturalmente, el relativo alto contenido de $\mathrm{Al}_{2} \mathrm{O}_{3}$, asegura la riqueza del clínker en $\mathrm{C}_{3} \mathrm{~A}$. Este alto contenido de $C_{3} A$ es bueno para las resistencias iniciales pero el cemento tiene un alto calor de hidratación y pobre resistencia al ataque de sulfatos.

Tener en cuenta que el comportamiento con el agua y la formación de los productos de hidratación resultantes de cada una de las fases anhidras, individual o por combinación, es suficientemente bien conocido para que muchas de las características -evolución del calor, velocidad de ganancia de resistencias, resistencia química, etc.- puedan ser previstas, al menos aproximadamente. Estas consideraciones sugieren que la extensión que existe para alterar el balance mineralógico de este tipo de clínker sea bastante limitado.

Una gran cantidad de energía requerida en la clinkerización está asociada con la descarbonatación del $\mathrm{CaCO}_{3}$. Si la cal (diferenciada del $\mathrm{CaCO}_{3}$ ) pudiera ser añadida a la mezcla se podrían conseguir sustanciales ahorros de energía. Kondo (2) estudió mezclas de crudos de materiales de cemento y escorias de horno que tienen la composición global mostrada en la tabla 4.

Después de la cocción, las muestras contenían $\mathrm{C}_{3} \mathrm{~S}, \mathrm{C}_{2} \mathrm{~S}, \mathrm{C}_{3} \mathrm{~A}$ y $\mathrm{C}_{4} \mathrm{AF}$. A pesar de la similitud en las composiciones química y mineralógica entre este y un OPC convencional hecho enteramente con materiales naturales, el clínker que incorporó escoria tenía un desarrollo de resistencia relativamente pobre. La razón de esto no está clara, y este proceso requiere claramente más estudio, si se va a realizar una incorporación de materiales crudos parcialmente descarbonatados en las hornadas de clínker.

Se ha desarrollado un material cementicio de

\section{POTENTIAL FOR THE DEVELOPMENT OF LOW ENERGY CEMENTS}

One way of removing the restrictive conditions is to change the clinker composition more drastically, so that at the highest clinkering temperatures both $\mathrm{C}_{3} \mathrm{~S}$ and $\mathrm{C}_{2} \mathrm{~S}$ are no longer in equilibrium with a melt phase. This removes the condition of isothermal invariancy imposed by conventional compositions This can be achieved in different ways; for example, some Ferrari cements are $\mathrm{C}_{2} \mathrm{~S}$-free and would appear to meet this condition. Table 3 shows a composition which, after cooling, consists mainly of $C_{3} S, C_{4} A F$ and $C_{3} A$. At clinkering temperatures $>1.350^{\circ} \mathrm{C}, \mathrm{C}_{3} S$ is probably the only crystalline phase which coexists stably in contact with melt. Of course, the relatively high content of $\mathrm{Al}_{2} \mathrm{O}_{3}$ ensures that the clinker is rich in $C_{3} A$. The high $C_{3} A$ content is good for the early strength, but the cement has a high heat of hydration and poor resistance to sulphate attack.

Note that the behaviour towards water and performance of the hydrated products resulting from each of the anhydrous phases, singly or in combination, is sufficiently well known to enable many of the features -heat evolution, rate of strength gain, chemical resistance, etc. - to be predicted, at least approximately. These considerations suggest that the scope which exists for altering the mineralogical balance of this type of clinker is rather limited.

A surprisingly high proportion of the energy requirement for clinkering is associated with the decarbonation of $\mathrm{CaCO}_{3}$. If, therefore, lime (as distinct from $\mathrm{CaCO}_{3}$ ) can be added to the batch, substantial saving in energy might be possible. Kondo (2) reported on mixtures of cement raw materials and blast furnace slag having the bulk composition shown in Table 4.

After firing, the batch contained $C_{3} S, C_{2} S, C_{3} A$ and $C_{4} A F$. Despite the similarity in chemical and mineralogical compositions between this and conventional OPC made entirely from natural material, the clinker incorporating slag had relatively poor strength development. The reasons for this are not clear, and this process clearly requires more study if the potential for incorporating partially decarbonated raw materials in clinker batches is to be realized.

A low-energy cementitious material has been developed from basic oxygen slags. These slags, unlike blast furnace slags, cannot be quenched to yield a glass and the crystalline phases obtained by either normal or forced cooling are not significantly cementitious. However, the slags are normally at a very high temperature and are capable of dissolving 
baja energía a partir de escorias básicas. Dichas escorias, contrariamente a las escorias de alto horno no pueden ser templadas para producir un vidrio y las fases cristalinas obtenidas, tanto por enfriamiento normal como por enfriamiento forzado, no son significativamente cementantes. Sin embargo, a muy altas temperaturas las escorias son capaces de disolver, apreciables cantidades de otros óxidos en estado fundido, sin que se produzca una cristalización.

Se ha desarrollado un aditivo "fritted" Ilamado "Camflux" que puede ser disuelto en la escoria todavía fundida. En la tabla 5 se muestra la composición del material resultante. La mezcla homogénea fundida puede enfriar con cristalización; mucho del óxido de hierro permanece en estado sólido. Esto unido al alto contenido en $\mathrm{MgO}$, produce durante el enfriamiento la cristalización de una fase con estructura de cloruro sódico ( $\mathrm{Ca}$, $\mathrm{Mg}$; Fe junto con $\mathrm{C}_{2} \mathrm{~S}$ y un ferrito). El producto resultante solamente es, en si mismo, débilmente cementante, pero es activado por el OPC.

Por otra parte un subproducto inútil se puede entonces convertir en una adición activa del cemento sin necesidad de un aporte sustancial de energía.

Otro camino hacia la obtención de cementos de baja energía es la producción de clínkeres ricos en $\mathrm{C}_{2} \mathrm{~S}$ (belita). El silicato bicálcico se clinkeriza antes que el $\mathrm{C}_{3} \mathrm{~S}$ : En cualquier caso el $\mathrm{C}_{3} \mathrm{~S}$ solamente es termodinámicamente estable a temperaturas por encima de aproximadamente $1.250^{\circ} \mathrm{C}$, de modo que la formación de $\mathrm{C}_{2} \mathrm{~S}$, en vez de la de $\mathrm{C}_{3} \mathrm{~S}$, se explica en términos termodinámicos en clínkeres de baja temperatura. Sin embargo la reactividad del $\mathrm{C}_{2} \mathrm{~S}$ respecto de la del $\mathrm{C}_{3} \mathrm{~S}$ es baja y se han hecho muchos esfuerzos en torno a la producción de $\mathrm{C}_{2} \mathrm{~S}$ reactivo, con el fin de mejorar la velocidad de adquisición de resistencias de los cementos ricos en $\mathrm{C}_{2} \mathrm{~S}$. El propio $\mathrm{C}_{2} \mathrm{~S}$ exhibe polimorfismo: Las fases $\alpha$ y $\alpha$, típicamente presentes a las temperaturas de clinkerización se transforman expontáneamente durante el enfriamiento. Dependiendo de las circunstancias, se puede obtener tanto la fase $\beta$ como la $\gamma$, de las que la última es bastante inerte frente a la hidratación. EI OPC normalmente contiene la fase $\beta$ del silicato bicálcico, aunque probablemente inestable respecto a la fase $\gamma$ a temperatura ambiente, persista debido a su estabilización cinética (i.e. la transformación a $\gamma$ está impedida) por la presencia de impurezas químicas. Por tanto un primer objetivo de los cementos de $\mathrm{C}_{2} \mathrm{~S}$ reactivo es obtener a temperatura ambiente el polimorfo más reactivo $\beta$. Habiendo obtenido $\beta-\mathrm{C}_{2} \mathrm{~S}$, su appreciable quantities of other oxides in the molten state without crystallization. A fritted additive termed 'Chamflux' has been developed which can be dissolved in the still-molten slag (3). The composition of the resulting mixed material is shown in Table 5. The homogeneous molten mixture is allowed to cool with crystallization; much of the iron oxide remains in the ferrous state. This, couple with the high $\mathrm{MgO}$ content, results in the crystallization of a sodium chloride-structured phase, (Ca, Mg, Fe) O, during cooling together with $\mathrm{C}_{2} \mathrm{~S}$ and a ferrite phase. The resulting product is itself only weakly cementitious but is activated by OPC. An otherwise useless byproduct can thus be converted into a useful cement blending agent without the need for substantial energy input.

Another route to low-energy cements lies in the production of clinkers rich in $\mathrm{C}_{2} \mathrm{~S}$ (belits). The dicalcium silicate phase is more readily clinkered than is $C_{3} S$ : in any event, $C_{3} S$ is thermodynamically stable only at temperatures above ca $1.250^{\circ} \mathrm{C}$, so that the formation of $\mathrm{C}_{2} \mathrm{~S}$, rather than $\mathrm{C}_{3} \mathrm{~S}$, in low-temperature clinkers is explicable on thermodynamic grounds. The reactivity of $\mathrm{C}_{2} \mathrm{~S}$ relative to $\mathrm{C}_{3} \mathrm{~S}$ is, however, low and much effort has gone into the production of reactive $\mathrm{C}_{2} \mathrm{~S}$ in order to improve the rate of strength gain of $\mathrm{C}_{2} \mathrm{~S}$-rich cements. $\mathrm{C}_{2} \mathrm{~S}$ itself exhibits polymorphism: the $\alpha$ and $\alpha$, phases, typically present at clinkering temperatures, transform spontaneously upon cooling. Depending on the circumstances, either $\beta$ or $\gamma$ phases may be obtained, of which the latter is rather unreactive towards hydration. OPC normally contains the $\beta$ phase of dicalcium silicate; although probably unstable with respect to $\gamma$ at ambient, it persists owing to its kinetic stabilization (i.e., the transformation to $\gamma$ is hindered) by the presence of chemical impurity. Therefore a primary goal of reactive $\mathrm{C}_{2} \mathrm{~S}$ cements is to obtain at ambient the more reactive $\beta$ polymorph. Having obtained $\beta-C_{2} S$, its reactivity may still vary, although the reasons for this are not clear. Low firing temperatures and rapid cooling of the $\beta$ product appear to enhance its reactivity. Thus, even preparations consisting wholly or mainly of $\beta-C_{2} S$ vary widely in their rate of strength gain. The reasons why $\beta-C_{2} S$ shoud exhibit such large variations in reactivity are unclear, and until they are, it is not possible systematically to improve the strength gain of belite-rich cements. Some of the factors believed to be responsible for controlling the reactivity of belite are listed in Table 6 .

The various polymorphs of $\mathrm{C}_{2} \mathrm{~S}$ are rather distantly related in structure except for $\alpha$ and $\alpha$ ' phases which are closely related. The transformation $\alpha^{\prime} \rightarrow \beta$ is probably semi- 
reactividad todavia puede variar, aunque las razones para ello no están claras. Parece que, unas temperaturas de cocción bajas y un enfriamiento rápido del $\beta$ obtenido, aumentan su reactividad. Entonces, aun preparaciones compuestas total o mayoritariamente de $\beta-\mathrm{C}_{2} \mathrm{~S}$ varian ampliamente en su velocidad de adquisición de resistencias. Las razones por las que el $\beta-C_{2} S$ exhibe tan grandes variaciones de reactividad no están claras, y hasta que lo estén, no es posible mejorar sistemáticamente la ganancia de resistencia de los cementos ricos en belita. En la tabla 6 están recogidos algunos de los factores, que se cree, son responsables del control de la reactividad de la belita.

Las diversas estructuras polimórficas del $\mathrm{C}_{2} \mathrm{~S}$ son diferentes entre sí, con la excepción de las $\alpha$ y $\alpha^{\prime}$ que son muy similares. La transformación $\alpha^{\prime} \rightarrow \beta$ es probab!emente semi-reconstructiva, ya que los cristales de la fase $\beta$ durante la inversión sufren un extenso maclado y normalmente a temperatura

TABLA 1

Resumen de temas relevantes en la investigación del cemento

1. Economicos en la producción y uso del cemento

2. Desarrollo de cementos de baja energía.

3. Papel de los halógenos en el proceso de clinkerización.

4. Cementos con adiciones activas.

5. Durabilidad de cementos y cementos con adiciones activas.

TABLA 2

Vías para los cementos de baja energía

\begin{tabular}{|l|l|}
\hline \multicolumn{1}{|c|}{ Tentativas } & \multicolumn{1}{|c|}{ Ejemplos } \\
\hline $\begin{array}{l}\text { Desarrollo de cementos } \\
\text { con composiciones } \\
\text { químicas y mineralógicas } \\
\text { distintas de las del OPC. }\end{array}$ & $\begin{array}{l}\text { Cementos con altos } \\
\text { contenidos en } \mathrm{Al}_{2} \mathrm{O}_{3} \mathrm{y} \\
\mathrm{Fe}_{2} \mathrm{O}_{3}, \text { cementos ricos } \\
\text { en sulfatos, etc. }\end{array}$ \\
$\begin{array}{l}\text { Desarrollo de cementos } \\
\text { similares al OPC, pero } \\
\text { que requieran menor } \\
\text { energía de } \\
\text { calentamiento. }\end{array}$ & $\begin{array}{l}\text { Clínkeres de alto } \\
\text { contenido en fase } \\
\text { belítica reactiva. }\end{array}$ \\
\hline
\end{tabular}

reconstructive, as crystals of the $\beta$ phase at ambient are twin extensively during inversion and nomally consist at ambient of mixtures of twinned polytypes. This is similar to the state of $C_{3} S$ crystals at ambient, although in the latter phase numerous minor polymorphic variations attending the transformation from rhombohedral to monoclinic to triclinic add further complications. Nevertheless, the microstructures of real crystals of both $\mathrm{C}_{2} \mathrm{~S}$ and $C_{3} S$ are complicated by impurity content and thermal history. In assessing the relative importance of these factors to the reactivity of the product, it has not been practicable to separate the contribution which each makes to the reactivity. Clearly, it is important to do so, as the empirical evidence suggests that the reactivity of $\beta-C_{2} S$ preparations can vary widely. Investigations of the reactivity of the substrate solid are further complicated by the fact that the accumulation of the solid products of hydration, their nature, thickness, adherence to the substrate, and diffusional properties may be the rate-determining step effectively controlling the reactivity of $\mathrm{C}_{2} \mathrm{~S}$.

Another development arising from belite-rich compositions has been the investigation of sulphate-rich compositions, containing principally $\beta-C_{2} S$ and the alumino-sulphate phase, $C_{4} A_{3} S^{-}$. The aluminosulphate phase has often been associated with expansive

\section{TABLE}

Summary of themes relevant to Cement Research

1. Economics of Cement Making and Use.

2. Potential for the Development of Low-Energy Cements.

3. Role of Halogens in Cement Clinkering.

4. Blended Cements.

5. Durability of Cements and Blended Cements.

\section{TABLE 2}

Routes to Low Energy Cement

\begin{tabular}{|l|l|}
\hline \multicolumn{1}{|c|}{ Approach } & \multicolumn{1}{c|}{ Examples } \\
\hline $\begin{array}{l}\text { Develop cements having } \\
\text { chemical and } \\
\text { mineralogical } \\
\text { compositions different } \\
\text { from OPC. }\end{array}$ & $\begin{array}{l}\text { Cements containing high } \\
\mathrm{Al}_{2} \mathrm{O}_{3} \text { or } \mathrm{Fe}_{2} \mathrm{O}_{3} \\
\text { contents, sulphaterich } \\
\text { cements, etc. }\end{array}$ \\
$\begin{array}{l}\text { Develop cements closely } \\
\text { related to OPC but } \\
\text { requiring less energy } \\
\text { to burn. }\end{array}$ & $\begin{array}{l}\mathrm{High} \mathrm{C}_{2} \mathrm{~S} \text { clinkers } \\
\text { containing reactive belite } \\
\text { phase. }\end{array}$ \\
\hline
\end{tabular}




\begin{tabular}{|c|c|}
\hline (Continuación) & \\
\hline $\begin{array}{l}\text { Formulaciones de OPC } \\
\text { pero asistidas por } \\
\text { reacciones cinéticas. }\end{array}$ & $\begin{array}{l}\text { Desarrollo de sistemas } \\
\text { de fundentes y } \\
\text { mineralizadores, por } \\
\text { ejemplo el floururo. }\end{array}$ \\
\hline $\begin{array}{l}\text { Cementos con adiciones } \\
\text { de otros materiales } \\
\text { potencialmente } \\
\text { conglomerantes. }\end{array}$ & $\begin{array}{l}\text { Uso de PFA, humo de } \\
\text { sílice, puzolanas } \\
\text { naturales, escorias } \\
\text { de hono como agentes } \\
\text { adicionales al OPC. }\end{array}$ \\
\hline
\end{tabular}

TABLA 3

Composición de cemento Ferrari de alto contenido de hierro

\begin{tabular}{|c|c|}
\hline Componente & Peso (\%) \\
\hline $\mathrm{CaO}$ & 64.6 \\
$\mathrm{SiO}_{2}$ & 16.9 \\
$\mathrm{Al}_{2} \mathrm{O}_{3}$ & 8.2 \\
$\mathrm{Fe}_{2} \mathrm{O}_{3}$ & 6.0 \\
$\mathrm{SO}_{3}$ & 1.5 \\
\hline
\end{tabular}

TABLA 4

Composición de un clínker con adiciones de escoria

\begin{tabular}{|c|c|}
\hline Componente & Peso (\%) \\
\hline $\mathrm{CaO}$ & 63.2 \\
$\mathrm{SiO}_{2}$ & 19.7 \\
$\mathrm{Al}_{2} \mathrm{O}_{3}$ & 5.1 \\
$\mathrm{Fe}_{2} \mathrm{O}_{3}$ & 4.2 \\
$\mathrm{SO}_{3}$ & 2.1 \\
\hline
\end{tabular}

TABLA 5

Material hidráulico basado en escorias "oxigen converter"

\begin{tabular}{|l|c|}
\hline Componente & Peso (\%) \\
\hline $\mathrm{CaO}$ & 36 \\
$\mathrm{SiO}_{2}$ & 10 \\
$\mathrm{Al}_{2} \mathrm{O}_{3}$ & 7 \\
$\mathrm{MgO}^{\text {Iron Oxide }}$ & 13 \\
$\mathrm{SO}_{3}$ & 34 \\
\hline
\end{tabular}

\begin{tabular}{|c|c|}
\hline (Continuación) & \\
\hline $\begin{array}{l}\text { Use existing } \\
\text { formulations for OPC, } \\
\text { but assist reaction } \\
\text { kinetics. }\end{array}$ & $\begin{array}{l}\text { Develop fluxed and } \\
\text { mineralized systems, } \\
\text { e.v.g. fluorine-assisted } \\
\text { burning. }\end{array}$ \\
\hline $\begin{array}{l}\text { Conserve cement by } \\
\text { admixtures with other } \\
\text { potentially-cementitious } \\
\text { materials. }\end{array}$ & $\begin{array}{l}\text { Use of PFA, silica fume, } \\
\text { natural pozzolans and } \\
\text { blast furnace slags as } \\
\text { blending agents with } \\
\text { OPC. }\end{array}$ \\
\hline
\end{tabular}

TABLE 3

\section{Composition of High-Iron Ferrari Cement}

\begin{tabular}{|c|c|}
\hline Component & Wt. (\%) \\
\hline $\mathrm{CaO}$ & 64.6 \\
$\mathrm{SiO}_{2}$ & 16.9 \\
$\mathrm{Al}_{2} \mathrm{O}_{3}$ & 8.2 \\
$\mathrm{Fe}_{2} \mathrm{O}_{3}$ & 6.0 \\
$\mathrm{SO}_{3}$ & 1.5 \\
\hline
\end{tabular}

TABLE 4

Composition of a Clinker Incorporating Slag

\begin{tabular}{|c|c|}
\hline Comoponent & Wt. (\%) \\
\hline $\mathrm{CaO}$ & 63.2 \\
$\mathrm{SiO}_{2}$ & 19.7 \\
$\mathrm{Al}_{2} \mathrm{O}_{3}$ & 5.1 \\
$\mathrm{Fe}_{2} \mathrm{O}_{3}$ & 4.2 \\
$\mathrm{SO}_{3}$ & 2.1 \\
\hline
\end{tabular}

TABLE 5

Hydraulic Material Based on Oxygen Converter Slag

\begin{tabular}{|l|c|}
\hline Component & Wt. (\%) \\
\hline $\mathrm{CaO}$ & 36 \\
$\mathrm{SiO}_{2}$ & 10 \\
$\mathrm{Al}_{2} \mathrm{O}_{3}$ & 7 \\
$\mathrm{MgO}$ & 13 \\
Iron Oxide & 34 \\
$\mathrm{SO}_{3}$ & 0 \\
\hline
\end{tabular}

MATERIALES DE CONSTRUCCION. Vol. 36, n.o 201, enero/febrero/marzo 1986 
reactividad todavia puede variar, aunque las razones para ello no están claras. Parece que, unas temperaturas de cocción bajas y un enfriamiento rápido del $\beta$ obtenido, aumentan su reactividad. Entonces, aun preparaciones compuestas total o mayoritariamente de $\beta-C_{2} S$ varían ampliamente en su velocidad de adquisición de resistencias. Las razones por las que el $\beta-\mathrm{C}_{2} \mathrm{~S}$ exhibe tan grandes variaciones de reactividad no están claras, y hasta que lo estén, no es posible mejorar sistemáticamente la ganancia de resistencia de los cementos ricos en belita. En la tabla 6 están recogidos algunos de los factores, que se cree, son responsables del control de la reactividad de la belita.

Las diversas estructuras polimórficas del $\mathrm{C}_{2} \mathrm{~S}$ son diferentes entre sí, con la excepción de las $\alpha$ y $\alpha^{\prime}$ que son muy similares. La transformación $\alpha^{\prime} \rightarrow \beta$ es probab!emente semi-reconstructiva, ya que los cristales de la fase $\beta$ durante la inversión sufren un extenso maclado y normalmente a temperatura

TABLA 1

Resumen de temas relevantes en la investigación del cemento

1. Economicos en la producción y uso del cemento

2. Desarrollo de cementos de baja energía.

3. Papel de los halógenos en el proceso de clinkerización.

4. Cementos con adiciones activas.

5. Durabilidad de cementos y cementos con adiciones activas.

\section{TABLA 2}

Vías para los cementos de baja energía

\begin{tabular}{|l|l|}
\hline \multicolumn{1}{|c|}{ Tentativas } & \multicolumn{1}{|c|}{ Ejemplos } \\
\hline $\begin{array}{l}\text { Desarrollo de cementos } \\
\text { con composiciones } \\
\text { químicas y mineralógicas } \\
\text { distintas de las del OPC. }\end{array}$ & $\begin{array}{l}\text { Cementos con altos } \\
\text { contenidos en } \mathrm{Al}_{2} \mathrm{O}_{3} \mathrm{y} \\
\mathrm{Fe}_{2} \mathrm{O}_{3}, \text { cementos ricos } \\
\text { en sulfatos, etc. }\end{array}$ \\
$\begin{array}{l}\text { Desarrollo de cementos } \\
\text { similares al OPC, pero } \\
\text { que requieran menor } \\
\text { energía de } \\
\text { calentamiento. }\end{array}$ & $\begin{array}{l}\text { Clínkeres de alto } \\
\text { contenido en fase } \\
\text { belítica reactiva. }\end{array}$ \\
\hline
\end{tabular}

MATERIALES DE CONSTRUCCION. Vol. 36, n.o 201, enero/febrero/marzo 1986 reconstructive, as crystals of the $\beta$ phase at ambient are twin extensively during inversion and nomally consist at ambient of mixtures of twinned polytypes. This is similar to the state of $\mathrm{C}_{3} \mathrm{~S}$ crystals at ambient, although in the latter phase numerous minor polymorphic variations attending the transformation from rhombohedral to monoclinic to triclinic add further complications. Nevertheless, the microstructures of real crystals of both $\mathrm{C}_{2} \mathrm{~S}$ and $C_{3} S$ are complicated by impurity content and thermal history. In assessing the relative importance of these factors to the reactivity of the product, it has not been practicable to separate the contribution which each makes to the reactivity. Clearly, it is important to do so, as the empirical evidence suggests that the reactivity of $\beta-C_{2} S$ preparations can vary widely. Investigations of the reactivity of the substrate solid are further complicated by the fact that the accumulation of the solid products of hydration, their nature, thickness, adherence to the substrate, and diffusional properties may be the rate-determining step effectively controlling the reactivity of $\mathrm{C}_{2} \mathrm{~S}$.

Another development arising from belite-rich compositions has been the investigation of sulphate-rich compositions, containing principally $\beta-C_{2} S$ and the alumino-sulphate phase, $C_{4} A_{3} S^{-}$. The aluminosulphate phase has often been associated with expansive

\section{TABLE 1}

Summary of themes relevant to Cement Research

1. Economics of Cement Making and Use.

2. Potential for the Development of Low-Energy Cements.

3. Role of Halogens in Cement Clinkering.

4. Blended Cements.

5. Durability of Cements and Blended Cements.

TABLE 2

Routes to Low Energy Cement

\begin{tabular}{|l|l|}
\hline \multicolumn{1}{|c|}{ Approach } & \multicolumn{1}{|c|}{ Examples } \\
\hline $\begin{array}{l}\text { Develop cements having } \\
\text { chemical and } \\
\text { mineralogical } \\
\text { compositions different } \\
\text { from OPC. }\end{array}$ & $\begin{array}{l}\mathrm{Cements} \mathrm{containing} \mathrm{high}_{\mathrm{Al}_{2} \mathrm{O}_{3} \text { or } \mathrm{Fe} \mathrm{O}_{3}} \mathrm{O}_{3} \\
\text { contents, sulphaterich } \\
\text { cements, etc. }\end{array}$ \\
$\begin{array}{l}\text { Develop cements closely } \\
\text { related to OPC but } \\
\text { requiring less energy } \\
\text { to burn. }\end{array}$ & $\begin{array}{l}\text { High } \mathrm{C}_{2} \mathrm{~S} \text { clinkers } \\
\text { containing reactive belite } \\
\text { phase. }\end{array}$ \\
\hline
\end{tabular}




\section{(Continuación)}

Formulaciones de OPC pero asistidas por

reacciones cinéticas.

Cementos con adiciones de otros materiales potencialmente conglomerantes.
Desarrollo de sistemas de fundentes $y$

mineralizadores, por ejemplo el floururo.

Uso de PFA, humo de sílice, puzolanas naturales, escorias

de hono como agentes adicionales al OPC.

TABLA 3

Composición de cemento Ferrari de alto contenido de hierro

\begin{tabular}{|c|c|}
\hline Componente & Peso (\%) \\
\hline $\mathrm{CaO}$ & 64.6 \\
$\mathrm{SiO}_{2}$ & 16.9 \\
$\mathrm{Al}_{2} \mathrm{O}_{3}$ & 8.2 \\
$\mathrm{Fe}_{2} \mathrm{O}_{3}$ & 6.0 \\
$\mathrm{SO}_{3}$ & 1.5 \\
\hline
\end{tabular}

TABLA 4

Composición de un clínker con adiciones de escoria

\begin{tabular}{|c|c|}
\hline Componente & Peso (\%) \\
\hline $\mathrm{CaO}$ & 63.2 \\
$\mathrm{SiO}_{2}$ & 19.7 \\
$\mathrm{Al}_{2} \mathrm{O}_{3}$ & 5.1 \\
$\mathrm{Fe}_{2} \mathrm{O}_{3}$ & 4.2 \\
$\mathrm{SO}_{3}$ & 2.1 \\
\hline
\end{tabular}

TABLA 5

Material hidráulico basado en escorias "oxigen converter"

\begin{tabular}{|l|c|}
\hline Componente & Peso (\%) \\
\hline $\mathrm{CaO}$ & 36 \\
$\mathrm{SiO}_{2}$ & 10 \\
$\mathrm{Al}_{2} \mathrm{O}_{3}$ & 7 \\
$\mathrm{MgO}$ & 13 \\
Iron Oxide & 34 \\
$\mathrm{SO}_{3}$ & 0 \\
\hline
\end{tabular}

\begin{tabular}{|l|l|}
\hline (Continuación) & $\begin{array}{l}\text { Use existing } \\
\text { formulations for OPC, } \\
\text { but assist reaction } \\
\text { kinetics. }\end{array}$ \\
$\begin{array}{l}\text { Conserve cement by fluxed and } \\
\text { mineralized systems, } \\
\text { e.v.g. fluorine-assisted } \\
\text { burning. } \\
\text { potentially-cementitious } \\
\text { materials. }\end{array}$ & $\begin{array}{l}\text { Use of PFA, silica fume, } \\
\text { natural pozzolans and } \\
\text { blast furnace slags as } \\
\text { blending agents with } \\
\text { OPC. }\end{array}$ \\
\hline
\end{tabular}

TABLE 3

Composition of High-Iron Ferrari Cement

\begin{tabular}{|c|c|}
\hline Component & Wt. (\%) \\
\hline $\mathrm{CaO}$ & 64.6 \\
$\mathrm{SiO}_{2}$ & 16.9 \\
$\mathrm{Al}_{2} \mathrm{O}_{3}$ & 8.2 \\
$\mathrm{Fe}_{2} \mathrm{O}_{3}$ & 6.0 \\
$\mathrm{SO}_{3}$ & 1.5 \\
\hline
\end{tabular}

TABLE 4

Composition of a Clinker Incorporating Slag

\begin{tabular}{|c|c|}
\hline Comoponent & Wt. (\%) \\
\hline $\mathrm{CaO}$ & 63.2 \\
$\mathrm{SiO}_{2}$ & 19.7 \\
$\mathrm{Al}_{2} \mathrm{O}_{3}$ & 5.1 \\
$\mathrm{Fe}_{2} \mathrm{O}_{3}$ & 4.2 \\
$\mathrm{SO}_{3}$ & 2.1 \\
\hline
\end{tabular}

TABLE 5

Hydraulic Material Based on Oxygen Converter Slag

\begin{tabular}{|l|c|}
\hline Component & Wt. (\%) \\
\hline $\mathrm{CaO}$ & 36 \\
$\mathrm{SiO}_{2}$ & 10 \\
$\mathrm{Al}_{2} \mathrm{O}_{3}$ & 7 \\
$\mathrm{MgO}$ & 13 \\
Iron Oxide & 34 \\
$\mathrm{SO}_{3}$ & 0 \\
\hline
\end{tabular}


TABLA 6

Factores controlantes de la reactividad intrínseca de los silicatos cálcicos

- ¿Pequeños cambios polimórficos afectan significativamente la reactividad de los silicatos cálcicos?

- ¿Excesos de energía contribuyen significativamente a la reactividad?

- ¿Las sustituciones químicas alteran la reactividad de los cristales de un silicato cálcico determinado?

- ¿La citada variable puede ser aislada para su estudio?

- ¿Hasta qué punto la reactividad está controlada por factores no relacionados con los anteriores, y sin embargo está controlada, por ejemplo, por barreras de difusión de productos sólidos de hidratación?

TABLA 7

Clinkeres no expansivos, ricos en sulfatos

\begin{tabular}{|c|c|}
\hline Componente & Peso (\%) \\
\hline $\mathrm{CaO}$ & $50-54$ \\
$\mathrm{SiO}_{2}$ & $21-24$ \\
$\mathrm{Al}_{2} \mathrm{O}_{3}$ & $15-20$ \\
$\mathrm{Fe}_{2} \mathrm{O}_{3}$ & $1-6$ \\
$\mathrm{SO}_{3}$ & $3-5$ \\
\hline
\end{tabular}

TABLA 8

Clínkeres de Alinita

\begin{tabular}{|l|c|}
\hline Componente & Peso (\%) \\
\hline $\mathrm{CaO}$ & $30-42$ \\
$\mathrm{SiO}_{2}$ & $12.75-17.5$ \\
$\mathrm{Al}_{2} \mathrm{O}_{3}$ & $1.6-12.4$ \\
$\mathrm{Fe}_{2} \mathrm{O}_{3}$ & $0.6-4.4$ \\
$\mathrm{SO}_{3}+\mathrm{CaCl}_{2}$ & $6-20$ \\
\hline
\end{tabular}

TABLA 9

Algunos agentes utilizados comúnmente como adiciones

\begin{tabular}{|l|l|l|}
\hline $\begin{array}{c}\text { Adicio- } \\
\text { nes }\end{array}$ & $\begin{array}{c}\text { Homogeneidad y } \\
\text { Naturaleza }\end{array}$ & \multicolumn{1}{|c|}{ Reactividad } \\
\hline $\begin{array}{l}\text { Humo } \\
\text { de si- } \\
\text { liice }\end{array}$ & $\begin{array}{l}\text { Homogéneos; alta } \\
\text { superficie } \\
\text { específica; vítrea } \\
\text { contenido de } \\
\text { sílice } \geq 95 \% .\end{array}$ & $\begin{array}{l}\text { Activada por } \\
\mathrm{Ca}(\mathrm{OH})_{2} \text { y bases } \\
\text { fuertes. Muy } \\
\text { reactiva. }\end{array}$ \\
\hline
\end{tabular}

.../...
TABLE 6

Factors Controlling the Intrinsic Reactivity of Calcium Silicates

- Do minor polymorphic changes affect significantly the reactivity of calcium silicates?

- Does strain energy contribute significantly to reactivity?

- Does chemical substitution alter the reactivity of crystals of a given calcium silicate?

- Can the separate variable cited above be isolated for study?

- To what extent is reactivity controlled by factors largely unrelated to the above, but is instead dominated (for example) by diffusion barriers of solid hydration products?

TABLE 7

Non-Expansive, Sulphate-Rich Clinkers

\begin{tabular}{|c|c|}
\hline Component & Wt. (\%) \\
\hline $\mathrm{CaO}$ & $50-54$ \\
$\mathrm{SiO}_{2}$ & $21-24$ \\
$\mathrm{Al}_{2} \mathrm{O}_{3}$ & $15-20$ \\
$\mathrm{Fe}_{2} \mathrm{O}_{3}$ & $1-6$ \\
$\mathrm{SO}_{3}$ & $3-5$ \\
\hline
\end{tabular}

TABLE 8

Alunite-Containing Clinkers

\begin{tabular}{|l|c|}
\hline Component & Wt. (\%) \\
\hline $\mathrm{CaO}$ & $30-42$ \\
$\mathrm{SiO}_{2}$ & $12.75-17.5$ \\
$\mathrm{Al}_{2} \mathrm{O}_{3}$ & $1.6-12.4$ \\
$\mathrm{Fe}_{2} \mathrm{O}_{3}$ & $0.6-4.4$ \\
$\mathrm{SO}_{3}+\mathrm{CaCl}_{2}$ & $6-20$ \\
\hline
\end{tabular}

TABLE 9

Some Commonly-Used Blending Agents

\begin{tabular}{|l|l|l|}
\hline $\begin{array}{l}\text { Blen- } \\
\text { ding } \\
\text { Agents }\end{array}$ & $\begin{array}{l}\text { Homogenity and } \\
\text { Nature. }\end{array}$ & Reactivity. \\
\hline $\begin{array}{l}\text { Silica } \\
\text { Fume }\end{array}$ & $\begin{array}{l}\text { Homogeneous, } \\
\text { High specific } \\
\text { surface. Glassy, } \\
\text { silica content } \geq \\
\geq 95 \% .\end{array}$ & $\begin{array}{l}\text { Activate by } \\
\mathrm{Ca}(\mathrm{OH})_{2} \text { and strong } \\
\text { bases. Very reactive. }\end{array}$ \\
\hline
\end{tabular}

.../... 


\section{(Continuación)}

\begin{tabular}{|c|c|c|}
\hline $\begin{array}{l}\text { Esco- } \\
\text { ria }\end{array}$ & $\begin{array}{l}\text { Homogénea. Baja } \\
\text { superficie vítrea, } \\
\text { en } 40 \% \mathrm{CaO} \sim \\
\sim 40 \% \mathrm{SiO}_{2} \text {. La } \\
\text { escoria de alto } \\
\text { horno contiene } \\
\text { poco } \mathrm{Fe} \text { y mucho } \\
\text { MgO. }\end{array}$ & $\begin{array}{l}\text { Activada por } \\
\mathrm{Ca}(\mathrm{OH})_{2} . A \text { menudo } \\
\text { molida separandose } \\
\text { o conjuntamente } \\
\text { para mejorar } \\
\text { reactividad. }\end{array}$ \\
\hline $\begin{array}{l}\text { Ceni- } \\
\text { zas } \\
\text { volan- } \\
\text { tes }\end{array}$ & $\begin{array}{l}\text { Cenizas volantes de } \\
\text { quemar carbón. } \\
\text { Heterogéneas, alta } \\
\text { superficie } \\
\text { específica. } \\
\text { La matriz vítrea } \\
\text { de aluminosilicatos } \\
\text { contiene mullita, } \\
\text { espinela, } \\
\text { cristobalita, etc. } \\
\text { (Clase F) o vidrio } \\
\text { cálcico conteniendo } \\
\text { melilita, etc. } \\
\text { (Clase C). }\end{array}$ & $\begin{array}{l}\text { Clase C } \\
\text { debilmente } \\
\text { cimentante. Ambas } \\
\text { clases } \mathrm{C} \text { y F } \\
\text { activadas por } \\
\mathrm{Ca}(\mathrm{OH})_{2} \text { y bases. } \\
\text { Matriz vitrea más } \\
\text { reactiva que las } \\
\text { fases cristalizadas. }\end{array}$ \\
\hline
\end{tabular}

ambiente consisten en mezclas de politipos maclados. Esto es semejante al estado de los cristales de $\mathrm{C}_{3} \mathrm{~S}$ a temperatura ambiente, aún cuando en este caso hay numerosas variaciones polimórficas menores que facilitan las transformaciones desde romboédrica a monoclínica y a triclínica añadiendo aún más complicaciones. Sin embargo, las microestructuras de los cristales tanto del $\mathrm{C}_{2} \mathrm{~S}$ como del $\mathrm{C}_{3} \mathrm{~S}$ son complicadas a causa de las impurezas que contienen y por su historia térmica. Evaluando la importancia relativa de estos factores con respecto a la reactividad del producto, no ha sido posible separar la contribución de cada uno sobre la reactividad. Evidentemente, es importante hacerlo, ya que las evidencias empíricas sugieren que la reactividad del $\beta-\mathrm{C}_{2} \mathrm{~S}$ puede variar mucho. Las investigaciones de la reactividad del sustrato sólido son muy complicadas, a causa de la acumulación de los productos de hidratación sólidos, su naturaleza, espesor, adherencia al sustrato y propiedades de difusión, que pueden ser controlantes de la reactividad del $\mathrm{C}_{2} \mathrm{~S}$.

Otros desarrollos que surgen de las composiciones ricas en belita son los correspondientes a las investigaciones de composiciones ricas en sulfato con contenido principal de $\beta-\mathrm{C}_{2} \mathrm{~S}$ y fase sulfoaluminato, $\mathrm{C}_{4} \mathrm{~A}_{3} \mathrm{~S}$. Esta fase ha sido a menudo asociada con los cementos expansivos, pero el bajo consumo energético de esas composiciones la incluye en el desarrollo de los clínkeres sin una expansión excesiva. La tabla 7 da unas composiciones típicas. En comparación con el OPC, esas composiciones tienen una relación $\mathrm{C} / \mathrm{S}$ baja, y contienen una proporción de $\mathrm{Al}_{2} \mathrm{O}_{3}$ y de $\mathrm{SO}_{3}$ sustancialmente más elevada. El $\mathrm{SO}_{3}$ queda retenido en el clínker a causa de

\section{(Continuación)}

\begin{tabular}{|c|c|c|}
\hline Slag. & $\begin{array}{l}\text { Homogeneous, Low } \\
\text { surface area Glassy. } \\
\text { Tipically, } 40 \% \\
\mathrm{CaO} \sim 40 \% \mathrm{SiO}_{2} \text {. } \\
\text { Blast furnace } \\
\text { slag contains little } \\
\text { Fe but much } \mathrm{MgO} \text {. }\end{array}$ & $\begin{array}{l}\text { Activated by } \\
\mathrm{Ca}(\mathrm{OH})_{2} \text { Often } \\
\text { ground separately } \\
\text { or interground to } \\
\text { enhance reactivity. }\end{array}$ \\
\hline PFA & $\begin{array}{l}\text { Fly ash from coal } \\
\text { burning } \\
\text { Inhomogeneous, } \\
\text { high specific } \\
\text { surface. } \\
\text { Aluminosilicate } \\
\text { glassy matrix } \\
\text { contains mullite, } \\
\text { spinel, cristobalite, } \\
\text { etc. (class F) or } \\
\text { calcic glass } \\
\text { containing melilite, } \\
\text { etc. (class C). }\end{array}$ & $\begin{array}{l}\text { Class } \mathrm{C} \text { weakly } \\
\text { cementitious. Both } \\
\text { class } \mathrm{C} \text { and } \mathrm{F} \\
\text { activated by } \\
\mathrm{Ca}(\mathrm{OH})_{2} \text { and } \\
\text { bases Glassy } \\
\text { matrix more reactive } \\
\text { than crystalline } \\
\text { phases. }\end{array}$ \\
\hline
\end{tabular}

cements, but the low-energy application of these compositions lies in development of clinkers which are not notably expansive. Table 7 shows typical ranges of compositions. Relative to OPC these compositions have a low $\mathrm{C} / \mathrm{S}$ ratio, and contain markedly more $\mathrm{Al}_{2} \mathrm{O}_{3}$ and $\mathrm{SO}_{3}$. The latter is retained at the comparatively low clinkering temperatures employed. The ferrite phase, abundant in OPC, is reduced or absent; depending on the $C / A$ ratio, either $C_{12} A_{7}$ or $C a$ may occur together with $\mathrm{C}_{4} \mathrm{~A}_{3} \mathrm{~S}^{-}$. The short-term strength of such cements is largely dependent on the nature and amount of the aluminate and aluminosulphathe phases present, while their strengths at longer ages are more critically dependent on the amount and reactivity of the belite phase. The importance of being able to predict and control belite reactivity, emphasized previously, is also a key factor in the future development of sulphate-rich cements. 
las bajas temperaturas de cocción empleadas. La fase ferrítica, que es abundante en el OPC, no existe o es muy escasa; dependiendo de la relación C/A tanto el $\mathrm{C}_{12} \mathrm{~A}_{7}$ como el $\mathrm{CA}$ pueden aparecer juntos con el $\mathrm{C}_{4} \mathrm{~A}_{3} \mathrm{~S}^{-}$. La resistencia a cortas edades de tales cementos depende en gran medida de la naturaleza y propoción de las fases aluminato y sulfoaluminato, mientras que las resistencias a edades más largas dependen de la cantidad y reactividad de la fase belítica. La importancia de la posibilidad de predecir y controlar la reactividad de la belita, puesto de relieve previamente, es también un factor clave en el futuro desarrollo de cementos ricos en sulfatos.

\section{PAPEL DE LOS HALOGENOS EN LA CLINKERIZACION}

Los iones procedentes de óxidos, solos o combinados como oxianiones, como por ejemplo los sulfatos, son las especies mayoritarias en el cemento. Sin embargo, estos iones pueden ser parcialmente sustituidos, en el sentido químico, por fluor o cloro. La carga iónica y el tamaño del ion cloruro $\left(\mathrm{Cl}^{-}\right)$son lo suficientemente diferentes de la carga y tamaño del ion $\mathrm{O}^{2-}$ que raramente es posible que el $\mathrm{Cl}^{-}$reemplace al $\mathrm{O}^{2-}$ en las estructuras cristalinas de los componentes del clínker anhidro. El fluor, sin embargo, es muy similar en tamaño al oxígeno; y las diferencias entre sus cargas iónicas pueden ser salvadas, con lo que el fluoruro puede perfectamente reemplazar al $\mathrm{O}^{2-}$.

Ya que el $\mathrm{Cl}^{-}$normalmente no sustituirá al $\mathrm{O}^{2-}$, su inclusión en el clínker alterará la mineralogía de este. En crudos con composición adecuada (Tabla 8 , tomada de 141) la fase alinita se puede desarrollar mediante tratamientos térmicos de relativamente baja temperatura, aproximadamente $1.250^{\circ} \mathrm{C}$. La alinita tiene una composición aproximada a $\mathrm{Ca}_{11}\left(\mathrm{Si}_{0.75} \mathrm{Al}_{0.25} \mathrm{~S}\right)_{4} \mathrm{O}_{18} \mathrm{Cl}$. Ese cloro es vital. Además se dice que adiciones de $\mathrm{Al}$ y $\mathrm{Fe}$ mejoran la producción y disminuyen las temperaturas de cocción.

La alinita es hidráulicamente activa y el proceso a simple vista parece atractivo. Sin embargo, la presencia de mucho ion cloruro sugiere la posibilidad de un aumento de la corrosión de las armaduras metálicas embebidas en hormigones hechas con cemento de alinita. Esta posibilidad junto con otros factores ha inhibido el desarrollo de composiciones conteniendo cloro. Estos factores incluyen el efecto corrosivo de los gases que contienen cloro sobre la propia planta de cemento, la posible contaminación

\section{ROLE OF HALOGENS IN CEMENT CLINKERING}

Oxide ions, either singly or combined as oxyanions, e.g. sulphate are the most abundant species in cement. But they can be partially replaced in the chemical sense by fluorine or chlorine. The ionic charge and size of chlorine as $\mathrm{Cl}^{-}$are sufficiently different from that of $\mathrm{O}^{2-}$ so that it is rarely possible for $\mathrm{Cl}^{-}$to replace $\mathrm{O}^{2-}$ in the crystal structures of anhydrous clinker minerals. Fluorine, on the other hand, is very similar in size to $\mathrm{O}^{2-}$; provided differences in charge can be overcome, fluorine may be able to substitute for $\mathrm{O}^{2-}$.

Since $\mathrm{Cl}^{-}$will not normally substitute for $\mathrm{O}^{2-}$ its inclusion in clinker batches has a decided effect on altering the mineralogy. In batches of appropriate composition [Table 8, taken from (4)], the alunite phase may be developed during comparatively low-temperature thermal treatments, typically $\sim 1.250^{\circ} \mathrm{C}$, Alunite itself has the approximate composition $\mathrm{Ca}_{11}\left(\mathrm{Si}_{0.75} \mathrm{Al} \mathrm{I}_{0.25} \mathrm{~S}\right)_{4} \mathrm{O}_{18} \mathrm{Cl}$.

This clorine is essential. Addition of $\mathrm{Al}$ and $\mathrm{Fe}$ is said to improve the yield and lower firing temperatures. alunite is cementitious, and the process seems superficially attractive. However, the presence of much chloride ion suggests the possibility than enhanced corrosion of steel reinforcements embedded in alunite cement concretes could occur. This possibility, in conjunction with several others, has inhibited development of chloride-containing compositions. Other unfavourable factors include the corrosive effect of chloride contained in the vapour phase on cement-burning plant, possible environmental contamination arising from vapour-phase losses of chlorine during burning and the rather varible strengths associated with the fired product.

Fluorine is less likely than $\mathrm{Cl}^{-}$to create 
ambiental por la presencia de cloro en los gases de salida y la alta variación en las resistencias asociadas al producto calcinado.

El fluoruro, probablemente, crea menos problemas que el $\mathrm{Cl}^{-}$en lo que a la corrosión de armaduras respecta ya que no parece que el $\mathrm{F}^{-}$altere significativamente el potencial de corrosión. Sin embargo, todavía hay problemas asociados al uso del fluor en la clinkerización del cemento; contaminación ambiental y ataques al refractario del horno pueden causar estos problemas.

En cualquier caso, parece lógico pensar que si la adición de fluor fuera ventajosa, estos problemas podrían ser salvados. Al tratar de fijar las aplicaciones del fluoruro se distinguiría entre dos usos, que no necesariamente han de ser excluyentes entre si. En primer lugar, el fluor puede ser usado como fundente. Un fundente aumenta la velocidad de reacción sin alterar significativamente el equilibrio termodinámico de la reacción o reacciones. De este modo los óxidos de $\mathrm{Fe}$ y $\mathrm{Al}$ actúan como fundentes para la formación del $\mathrm{C}_{2} \mathrm{~S}$ y del $\mathrm{C}_{3} \mathrm{~S}$ durante la clinkerización convencional. Estos no alteran la estabilidad termodinámica del $\mathrm{C}_{3} \mathrm{~S}$ con respecto al $\mathrm{C}_{2} \mathrm{~S}$ o a la cal sino que en un rango apropiado de temperaturas facilitan el transporte másico de los iones $\mathrm{Ca}, \mathrm{Si}$ y $\mathrm{O}$ vía una fase líquida permitiendo por tanto que el $\mathrm{C}_{3} \mathrm{~S}$ se forme más rápidamente de lo que sería posible por reacciones en estado sólido. Por otra parte el fluoruro puede ser usado como mineralizador. Los mineralizadores promocionan la formación de una determinada fase aunque alterando su estabilidad termodinámica con respecto a otras fases menos favorecidas. Esto lleva consigo la participación en la reacción de alguna solución sólida en una o más fases cristalinas, generalmente (aunque no necesariamente) en la fase deseada, por ejemplo el $\mathrm{C}_{3} \mathrm{~S}$ en el clínker.

Aunque los dos términos, fundentes y mineralizador, se usan con frecuencia (incorrectamente) sin distinción, una comprensión sistemática de la acción de los componentes minoritarios durante la clinkerización, sólo se puede desarrollar si se mantiene esta diferencia.

La acción del fluor entra dentro de este contexto. Frecuentemente el fluoruro se ha añadido a crudos, calcinados posteriormente a temperaturas normales de clinkerización. Si el fluoruro queda retenido, entra en la fase líquida haciendo descender su viscosidad y aumentando su poder disolvente. También, la cantidad de líquido puede aumentar si se mantienen constantes el resto de las corrosion problems associated, with reinforcing steel in concrete, as $F^{-}$appears not to alter significantly the threshold potential for corrosion. There are, however, still problems associated with the use of $F$ in cement burning; environmental pollution and attack on kiln linings, may cause problems. However, it appears likely that if addition of fluorine were otherwise advantageous, these problems could be overcome. In assessing the applications of flurine, I distinguish between two uses, which are not necesarily mutually exclusive. Firstly, fluorine may be used as a flux. A flux uncreases the rate of reaction without significantly altering the thermodynamic equilibrium of the reaction or reactions. Thus $A l$ and Fe oxides act as fluxes for formation of $\mathrm{C}_{2} \mathrm{~S}$ and $\mathrm{C}_{3} \mathrm{~S}$ during conventional clinkering. They do not, for example, alter the thermodynamic solubility of $\mathrm{C}_{3} \mathrm{~S}$ relative to $\mathrm{C}_{2} \mathrm{~S}$ or $\mathrm{CaO}$. But in an appropriate temperature range, they facilitate mass transport of $\mathrm{Ca}, \mathrm{Si}$ and $\mathrm{O}$ ions via a liquid phase, thereby allowing $C_{3} S$ to form more rapidly than would be possible by solidstate reactions alone. Mineralizers, on the other hand, promote the formation of a selected phase by altering its thermodynamic stability relative to other less-favoured phases. This implies some solid solution in one or more of the crystalline phases participating in the reaction, usually (but not necessarily) in the desired phase, in typically $C_{3} S$ clinker. Although the two terms, flux and mineralizer, are often (but incorrectly) used without distinction, a systematic understanding of the action of minor components during clinkering can only be developed if this distintion is maintained.

The action of fluorine is a case in point. It has frequently been added to clinker batches subsequently fired at normal clinkering temperures. If retained, the fluorine enters the melt phase, decreasing its viscosity and increasing its solvent power: the amount of liquid may also be increased, other factors being equal. In this application, the fluorine is being used as a flux to assist burning. It must be recalled that as clinker is cooled, and crystallization occurs fluorine in the melt must enter into the crystal structure of one or more phases. Our present understanding of the behaviour of fluorine in conjunction with other oxide components of normal clinkers suggest that much of the $F$ will enter a fluoroaluminate phase based on solid solutions in the $C_{12} A_{7}-C_{11} A_{7} \cdot \mathrm{Ca} F_{2}$ series.

The group at Aberdeen have attempted to apply the principles of crystal chemistry, phase equilibria and crystal structure determination to eludicating problems associated with $F$ utilization: in this respect, I with to describe briefly the current status of work undertaken by myself with colleagues: two from Spain, $M$. 
variables. En este sentido, el fluoruro se usa como fundente para ayudar a la cocción.

Hay que poner incapié en que puesto que el clinker sufre un enfriamiento que dá lugar a una cristalización, el fluoruro en el líquido debe entrar en la estructura cristalina de una o más fases. El estado actual del conocimiento de la conducta del fluor junto con otros componentes normales en los clínkeres sugiere que una gran cantidad del $F$ entrará como una fase fluoraluminato basada en la serie de soluciones sólidas $C_{12} A_{7}-C_{11} A_{7} C a F_{2}$.

El grupo de Aberdeen ha intentado aplicar los principios de la cristalografía, del equilibrio de fases y de la determinación de estructuras cristalinas para resolver problemas asociados con la utilización del fluoruro: a este respecto, deseo describir brevemente el estado actual del trabajo llevado a cabo por mi mismo y por mis colegas: M. Pérez Méndez y José Fayos (España). E. Shame (Malawi).

La fase $\mathrm{C}_{3} \mathrm{~S}$, por si misma no es capaz de acomodar cantidades significativas de $\mathrm{F}^{-}$en solución sólida a menos que también se hagan otras sustituciones concordantes, la más importante de las cuales parece ser la sustitución de $\mathrm{Al}$ (también $\mathrm{Fe}$ ) por Si en posiciones tetraédricas, acompañada de sustituciones $\mathrm{F}^{-}$por $\mathrm{O}^{2-}$. Esto da lugar a series de soluciones sólidas del tipo

$\mathrm{Ca}_{3} \mathrm{Si}_{1-x} \mathrm{Al}_{\mathrm{x}} \mathrm{O}_{5-\mathrm{x}} \mathrm{F}_{\mathrm{x}}$. La estabilidad de las soluciones sólidas especialmente a bajas temperaturas se muestra en la Fig. 1. En ausencia de $\mathrm{F}^{-}$el $\mathrm{C}_{3} \mathrm{~S}$ no es estable por debajo de $1.250^{\circ} \mathrm{C}$ pero la doble sustitución $(\mathrm{Al}+\mathrm{F})$ por $(\mathrm{Si}+\mathrm{O})$ permite estabilizar soluciones sólidas de $\mathrm{C}_{3} \mathrm{~S}$ que se forman a temperaturas del orden de $1.075^{\circ} \mathrm{C}$. Las fases en equilibrio y los estudios cinéticos $(5,6)$ muestran que esto ocurre rápidamente incluso a temperaturas inferiores a las empleadas convencionalmente en la clinkerización. Es más, el producto final tiene una alta proporción de $\mathrm{C}_{3} \mathrm{~S}$ (aproximadamente $80-90 \%)$ y contiene poca cal libre. El fluor si está correctamente dosificado acaba entrando en el $\mathrm{C}_{3} \mathrm{~S}$ que se estabiliza a temperatura ambiente en su forma romboédrica, que es la más activa. Los iones sulfatos también pueden incorporarse en estas soluciones sólidas. Restringiendo la reacción a las temperaturas subsolidus, se fomenta la distribución del fluoruro en la fase sólida. Sin embargo si la temperatura de reacción se eleva hasta desarrollar una fase liquida, el fluor se distribuye entre las fases sólida y líquida. Puesto que la distribución favorece a la fase líquida, esto representa un uso del fluoruro menos eficaz, porque el líquido cristaliza posteriormente para producir fases que
Perez Mendez and Jose Fayos and one from Malawi, El Shame.

The $C_{3} S$ phase itself is not capable of accommodating significant quantities of $\mathrm{F}^{-}$in solid solution unless other concomitant substitutions are also made, the most important of which appear to be substitution of $\mathrm{Al}$ (also $\mathrm{Fe}$ ) for Si in tetrahedral sites, accompanied by $\mathrm{F}^{-}$for $\mathrm{O}^{2-}$ substitution. This gives rise to a series of solid solutions of the type $\mathrm{Ca}_{3} \mathrm{Si}_{1-x} \mathrm{Al}_{x} \mathrm{O}_{5-x} \mathrm{~F}_{x}$. The stability of the solid solutions especially at lower temperatures is shown in Fig. 1. In the absence of $\mathrm{F}^{-}, \mathrm{C}_{3}$ is not stable below $1.250^{\circ} \mathrm{C}$, but the double substitution $(A I+F)$ for $(\mathrm{Si}+\mathrm{O})$ permits stable $\mathrm{C}_{3} S$ solid solutions to form at temperatures down to $1.075^{\circ} \mathrm{C}$. Phase equilibrium and kinetic studies $(5,6)$ show that this occurs rapidly even at temperatures lower than those conventionally employed in clinkering. Furthermore, the product is high in $\mathrm{C}_{3} \mathrm{~S}$, typically $80-90 \%$, and contains little free lime. The $F$, if correctly proportioned, ends up entirely in the $C_{3} S$ which is stabilized to ambient as the more reactive rhombohedral phase. Sulphate igns may also be incorporated in these solid solutions. By restricting reaction to subsolidus temperatures, partition of fluorine into the solid phase is encouraged. However, if the reaction temperature rises such that a liquid phase develops, fluorine is partitioned between solid and liquid phases. Since the partition favours the liquid phase, this represents a less effective use of fluorine, because the liquid subsequently crystallizes to yield phases which contribute less to the cementitious potential of the system, relative to $\mathrm{C}_{3} \mathrm{~S}$.

The rules for proportioning fluorine are not completely understood for compositions where $F$ is in excess fo that required to saturate $C_{3} S$ solid solutions, or where melt formation occurs. Two solid F-containing phases have recently been characterized in our laboratory. The first is the socalled Tanaka's phase. Crystal structure determinations disclose that it has the type formula $\mathrm{Ca}_{6-0.5 x} \mathrm{Si}_{2} \mathrm{O}_{10-x} \mathrm{~F}_{x}$ and is closely related in structure to $\mathrm{C}_{3} \mathrm{~S}$, differing principally in the stacking of blocks of structure in the $c$ direction and including some $F$ for $O$ substitution in the stacking sequence (7).

Although closely related to $C_{3} S$ in its structure, it is not very reactive with water and, as a small amount of $F$ can combine with a large amount of $\mathrm{CaO}$ and $\mathrm{SiO}_{2}$ during its formation, it is viewed as constituting an undesirable phase. Furtunately it appears to develop during devitrification of a fluorine-rich melt, so its formation can probably be avoided. Where alkalis and sulphates are present, fluorine 
contribuyen menos que el $\mathrm{C}_{3} \mathrm{~S}$ al potencial aglomerante del sistema.

No se comprenden todavía las reglas que rigen la distribución del fluoruro en composiciones en las que el $F$ está en exceso con respecto a la cantidad requerida para saturar las soluciones sólidas de $\mathrm{C}_{3} \mathrm{~S}$, o en donde se dá la formación de un líquido. Recientemente se han caracterizado en nuestros laboratorios dos fases sólidas que contienen fluor. La primera es la llamada fase de Tanaka. Determinaciones de la estructura cristalina, revelan que esta fase tiene una fórmula del tipo $\mathrm{Ca}_{6-0.5 \mathrm{x}} \mathrm{Si}_{2} \mathrm{O}_{10-\mathrm{x}} \mathrm{F}_{\mathrm{x}}$ y está estrechamente relacionada a la estructura del $\mathrm{C}_{3} \mathrm{~S}$, diferenciándose principalmente en el empaquetamiento de bloques de estructura en la dirección $\mathrm{C}$ y en la inclusión de algo de fluor que sustituye a algunos oxígenos en la secuencia de empaquetamiento (7).

Aunque estrechamente relacionado con la estructura del $\mathrm{C}_{3} \mathrm{~S}$, este compuesto no es muy reactivo con el agua y además como una pequeña cantidad de $\mathrm{F}$ se puede combinar con una gran cantidad de $\mathrm{CaO}$ y $\mathrm{SiO}_{2}$, se considera a esta fase como un constituyente no deseable. Afortunadamente parece que se desarrolla durante la desvitrificación de un líquido rico en fluor así que probablemente se puede evitar su formación. Cuando álcalis y sulfatos están presentes, el fluoruro también se puede combinar para producir KF.2 $\mathrm{Ca}_{6}\left(\mathrm{SO}_{4}\right)\left(\mathrm{SiO}_{4}\right) \mathrm{O}$. La determinación de su estructura cristalina ha confirmado la fórmula y ha mostrado que ésta también está estrechamente relacionada con la del $\mathrm{C}_{3} \mathrm{~S}$. Las columnas de octaedros $\mathrm{Ca}-\mathrm{O}$ presentes en el $\mathrm{C}_{3} \mathrm{~S}$ están dislocadas para permitir la inclusión de poliedros $\mathrm{K}-\mathrm{O}$ y de anillos de tetraedros aislados de $\mathrm{SiO}_{4}{ }^{4-}$ presentes en el $\mathrm{C}_{3} \mathrm{~S}, 1 / 3$ de los tetraedros son reemplazados por $\mathrm{SO}_{4}{ }^{2-}$ en un orden determinado. La colocación final neta tiene una mayor estabilidad termodinámica a bajas temperaturas y se cree que es termodinámicamente estable por debajo de los $1.250^{\circ} \mathrm{C}$. La estructura completa junto con una clasificación cristaloquímica de otras fases de esta familia están a punto de ser públicas (8). Desafortunadamente, esta fase tiene mucha menos reactividad con el agua que el $\mathrm{C}_{3} \mathrm{~S}$.

Un reciente trabajo ha establecido la distribución del fluoruro entre las fases sólidas para una mejor comprensión. Varias fases nuevas hasta ahora desconocidas o pobremente caracterizadas han recibido una caracterización adecuada. Sin embargo, aún queda mucho trabajo por hacer. Es decir, puede ser que no todas las fases que may also be combined to yield KF.2 $\left(\mathrm{Ca}_{6}\left(\mathrm{SO}_{4}\right)\left(\mathrm{SiO}_{4}\right)_{2} \mathrm{O}\right]$. Determination of its crystal structure has confirmed the formula and shown that its structure also closely resembles that of $\mathrm{C}_{3} \mathrm{~S}$. The columns of $\mathrm{Ca}-\mathrm{O}$ octahedra present in $\mathrm{C}_{3} \mathrm{~S}$ are disrupted to allow for inclusion of K-O polyhedra and, of the strings of isolated $\mathrm{SiO}_{4}{ }^{4-}$ tetrahedra present in $\mathrm{C}_{3} \mathrm{~S}, 1 / 3$ of the tetrahedra are replaced on an ordered basis by $\mathrm{SO}_{4}{ }^{2-}$. The net arrangemet, while $\mathrm{C}_{3} \mathrm{~S}$-like, has greater thermodynamic stability at low temperatures and is believed to be thermodynamically stable below $1.250^{\circ} \mathrm{C}$. The complete structure together with a crystallochemical classification of other phases in this family are now in press (8). Unfortunately, this phase has much less reactivity towards water than $C_{3} S$.

Recent work has enabled the partition of fluorine amongst solid phases to be better understood. Several new phases, hitherto unknown or poorly characterized, have received adequate characterization. However, more work remains to be done. It is by no means certain that all the fluorine-containing phases have yet been discovered and the phase relations existing in fluorine modified systems must receive further study before the scientific basis for fluorine-assisted clinkering can be said to be established. 
contienen fluoruro hayan sido todavía descubiertas y las relaciones entre las fases de los sistemas modificados por el fluoruro deben ser objeto de estudio antes de establecer una base científica sobre clínkeres con fluor.

\section{CEMENTOS CON ADICIONES}

En el tiempo disponible no me es posible dar cuenta completa de las características más notables de los cementos de adición. Deseo, sin embargo, concentrar la exposición en unas pocas ideas generales, ya que están difundidas entre tanta información detallada, que, por otra parte, es indudablemente válida.

Las adiciones activas se utilizan porque en presencia de cemento reaccionan con agua para dar, como productos de reacción, compuestos que tienen propiedades hidráulicas - ligantes- por sí mismas. El producto de hidratación así obtenido es silicato cálcico hidratado, C-S-H, esencialmente idéntico al formado durante la hidratación del OPC, aunque con frecuencia tienen una relación $\mathrm{C} / \mathrm{S}$ diferente del portland sin adición $(1,7)$; en cementos con adición, las relaciones $\mathrm{C} / \mathrm{S}$ son bajas aproximadamente 0.9. Más adelante se destacarán otras diferencias.

Las adiciones activas deberán contener sílice en forma reactiva. Algunas se dan en la tabla 9. Se diferencian considerablemente por su homogeneidad y su naturaleza química.

Con poca cantidad $\left(\mathrm{S}^{2-} \simeq 10 \%\right.$ del peso del cemento) los efectos de las adiciones activas no son demasiado importantes pero a medida que aumenta su proporción respecto al cemento, el efecto que ejercen sobre el conjunto del sistema va incrementándose.

Esas diferencias son poco apreciables a cortas edades ya que la baja reactividad de las adiciones puede hacer que se comporten, en parte, como un árido fino, y tan solo a mayores edades cuando una parte considerable de la adición ha reaccionado, se ponen de relieve las diferencias existentes entre el OPC y el OPC con adiciones.

Esas diferencias químicas están siendo estudiadas en varios laboratorios, incluyendo el nuestro. Los cementos de humo de sílice, por ejemplo, reaccionan rápidamente y después de 8-12 meses de realizada la mezcla, a $18-20^{\circ} \mathrm{C}$ y $100 \%$ de humedad, muestran una reducción en el contenido de $\mathrm{Ca}(\mathrm{OH})_{2}$ que corresponde al consumo total de humo de $\mathrm{SiO}_{2}$, en relación con el OPC. La reducción en el contenido de $\mathrm{Ca}(\mathrm{OH})_{2}$ es

\section{BLENDED CEMENTS}

In the time available it is not possible to give a complete account of the features of blended cements. I would therefore like to concentrate on a few broad perspectives, because these are often lost sight of in the mass of highly detailed information which is becoming available.

Blending agents are useful because in the presence of cement, they react with the aqueous phase to give as reaction products materials which are cementitious in their own right. The normal cementitious product thus obtained is calcium silicate hydrate, C-S-H, essentially identical with that formed during the hydration of OPC, although often having a different $\mathrm{C} / \mathrm{S}$ ratio from that found in unmodified cement ( $~ 1.7$ ); in blended cements $\mathrm{C} / \mathrm{S}$ ratios range as low as $\sim 0.9$. Other differences will emerge.

The blending agents must therefore contain silica in a reactive form. Several sources of blending agents are tabulated in Table 9. These differ considerably in their homogenity and basic chemical makeup. At low levels (Ca $5-10 \%$ of the weight of cement) these differences do not appear too important but as the proportion relative to cement is increased, the chemistry of the modifier increasingly impresses itself upon the system as a whole. These differences may not be too apparent in the short term because less-reactive additives may, in the short term, behave partly as a fine aggregate and only as longer ages, when an appreciable fraction has reacted, differences between OPC and OPC-blends become more apparent. These chemical differences are being studied in several laboratories, including our own. Silica fume cements, for example, react rapidly and in 8-12 months well-mixed composites aged at $18-20^{\circ} \mathrm{C}$ and $100 \%$ humidity show a reduction in $\mathrm{Ca}(\mathrm{OH})_{2}$ content which corresponds to complete consumption of the $\mathrm{SiO}_{2}$ fume, relative to OPC. The reduction in $\mathrm{Ca}(\mathrm{OH})_{2}$ content is nearly proportional to the amount of silica fume added, some $20-25 \%$ by weight of silica fume being required to neutralize all the $\mathrm{Ca}(\mathrm{OH})_{2}$ of a normal OPC. The C-S-H phase in fully neutralized batches tends to have a low $\mathrm{C} / \mathrm{S}$ ratio. This low ratio $\mathrm{C}-\mathrm{S}-\mathrm{H}$ uptakes alkali, especially $K$, from the pore fluid with the result that the internal $\mathrm{pH}$ is typically one unit less than it would be in an unmodified cement. One 
aproximadamente, proporcional a la cantidad de humo de sílice añadido, sobre el 20-25\% en peso, que se necesita para neutralizar todo el $\mathrm{Ca}(\mathrm{OH})$, de un OPC normal. La fase C-S-H en disoluciones completamente neutralizadas tiende a tener una relación $\mathrm{C} / \mathrm{S}$ baja. Este C-S-H, de baja relación, capta álcalis, especialmemte $\mathrm{K}$, de la disolución que rellena los poros, con el resultado de que el $\mathrm{OH}$ interno es normalmente una unidad inferior a la que tendrá el cemento puro. Una unidad de $\mathrm{pH}$ corresponde a una disminución de 10 en el contenido en $\mathrm{OH}^{-}$.

Existen distintos pareceres acerca de si esa disminución de la alcalinidad rebajará la protección de las armaduras a la corrosión, que ofrece el OPC no modificado. Más adelante volveremos sobre estos.

La ceniza volante de clase $F$ probablemente se comportará a edades largas de forma similar a la del humo de sílice. Sin embargo es difícil demostrarlo a causa de su baja superficie específica, en comparación con la del humo de sílice. Los morteros PFAcementos de adición, envejecidos durante 14 meses a $18^{\circ} \mathrm{C}$ todavía presentan una gran capacidad de retención de $\mathrm{Ca}(\mathrm{OH})_{2}$ (9). Las cenizas volantes de clase $\mathrm{C}$ y las escorias, por otra parte, son relativamente ricas en cal y tienen potencialmente mucha menor capacidad de combinación con el $\mathrm{Ca}(\mathrm{OH})_{2}$. De hecho, las experimentaciones citadas en (9), de 14 meses de duración, demuestran que una escoria de alto horno fija una cantidad mínima del $\mathrm{Ca}(\mathrm{OH})_{2}$ suministrado por el OPC.

Hay, sin embargo, importantes hechos en el comportamiento químico de los cementos de adición, que han de ser investigados. Mientras que puede describirse tanto la evolución general del contenido de $\mathrm{Ca}(\mathrm{OH})_{2}$, como la cantidad y composición de las fases C-S-H, se conoce poco acerca de las fases AFm y AFt en los cementos de adición. Hay otras incógnitas que aún permanecen sin resolver; por ejemplo, ¿qué pasa con el $\mathrm{Mg}$ de los cementos de escoria? simplemente, no lo sabemos con seguridad. Además, los cementos de escoria en particular, introducen una brusca disminución de su potencial redox. La fuente de esta disminución de potencial, probablemente está ligada a un sustancial contenido (0.5-1.5\%) de azufre reducido presente en la escoria. Su destino durante la reacción escoria-cemento, es sin embargo, desconocido, queda todavía mucho trabajo por hacer en la química de los procesos de hidratación en presencia de grandes cantidades de adiciones activas, para poder conocerlas suficientemente.

Como respuesta a estos desafíos, se han
$\mathrm{pH}$ unit corresponds to a decrease by a factor of 10 in $\mathrm{OH}^{-}$content, and there is some controversy as to whether this reduction in alkalinity could decrease the corrosion protection afforded to steel by unmodified OPC. We return to this point. Class $F$ flyash will presumably bahave at long ages in the same way as silica fume. However, because of its low specific surface area relative to silica fume, it is difficult to prove this. Composite PFA-cement blends, aged for 14 months at $18^{\circ} \mathrm{C}$, still retain plentiful $\mathrm{Ca}(\mathrm{OH})_{2}(9)$.

Slags and class $C$ flyash, on the other hand, are relatively lime-rich and have much less potential for combining with $\mathrm{Ca}(\mathrm{OH})_{2}$. Certainly, experiments cited in (9), of 14 months duration show that a blast furnace slag makes minimal demand on the $\mathrm{Ca}(\mathrm{OH})_{2}$ content supplied by the OPC.

These are, however, important features in the chemical balance within these blended cements which requires investigation. While the general evolution of the $\mathrm{Ca}(\mathrm{OH})_{2}$ content and the amount and composition of the C-S-H phases can be outlined, less is known about the $A F_{m}$ and $A F_{t}$ phases in blended cements. Other mysteries remain: what, for example, becomes of the Mg content of slag cements? We simply do not know with certainty. Furthermore, slag cements in particular introduce a sharp lowering of the internal redox potential. The source of this lowered potential presumably lies in the substantial content (0.5-1.5 \%) of reduced sulphur present in the slag. Its fate during the slag-cement reaction is, however, largely unknown. Much work is thus required before the basic chemistry of the hydration processes in the presence of large amounts of blending agents can be said to be well-established.

In response to the challenges, several new methods habe been developed which enable the course of reaction in blended cements to be followed. For example, the chemical evolution of the composition of the pore fluid permeating cement paste can be samples directly by placing cylinders of set, matured paste of normal $\mathrm{w} / \mathrm{c}$ ratios in a powerful squeezer and analyzing the expressed pore fluid. The technique has been reviewed elsewhere (10). Fig. 2 shows the variations in pore fluid chemistry which can be expected. In these experiments $\mathrm{Ca} 200 \mathrm{~g}$ cylinders were made to $w / c$ ratios lying between 0.55 and 0.60 , aged for 14 months at $100 \% \mathrm{RH}$ and squeezed. The OPC used was low in sodium (0-0.19\% $\left.\mathrm{Na}_{2} \mathrm{O}\right)$. Fig. 2 shows two $\mathrm{Na}$ concentrations; the observed $\mathrm{Na}$ concentration of the pore fluid (ordinate) and the "expected" $\mathrm{Na}$ concentration. This latter is calculated from the mass balance of the system, on the assumption that all the $\mathrm{Na}$ is released to the pore fluid. If this condition is met, analyses 
desarrollado nuevos métodos que posibilitan seguir el curso de las reacciones en los cementos con adición. Por ejemplo, la evolución química de la composición del líquido de los poros en las pastas de cemento puede estudiarse directamente a partir de muestras de pastas, con relaciones agua/cemento normales, comprimiéndolas dentro de un molde adecuado por una prensa de gran capacidad, y analizando el líquido extraído. Esta técnica la hemos estudiado en (10). La fig. 2 muestra las variaciones químicas que pueden esperarse en el líquido de los poros. En esos ensayos se prepararon cilindros con aproximadamente $200 \mathrm{~g}$ de muestras con relaciones agua/cemento comprendidas entre 0.55 y 0.60 , envejecidas durante 14 meses, al $100 \%$ de H.R., y presentadas. EI OPC utilizado era bajo en sodio (0-0.19\% $\mathrm{Na}_{2} \mathrm{O}$ ). La fig. 2 señala dos concentraciones de $\mathrm{Na}$; la concentración de $\mathrm{Na}$ observada en el líquido de los poros (representada en las ordenadas del gráfico de la fig. 2), y la concentración de $\mathrm{Na}$ "esperada". Esta última está calculada a partir del balance de masas del sistema, considerando que todo el $\mathrm{Na}$ está en el líquido de los poros. Si se da esta condición, los análisis deberán estar sobre la línea dibujada con trazos. Sin embargo, el OPC da valores muy por debajo de aquella linea, lo que indica que una gran parte del $\mathrm{Na}$ está retenido en los productos sólidos de la hidratación. Los análisis de microscopía electrónica revelan que la fase C-S-H contiene mucho $\mathrm{Na}$, probablemente reemplazando al $\mathrm{Ca}$. Se da también el efecto del $15 \%$ de la adición activa. El humo de sílice (círculo relleno; en el gráfico) no solamente contiene poco $\mathrm{Na}$, sino que aumenta sustancialmente la capacidad de absorción de $\mathrm{Na}$ por el sistema. La sílice de "flame hidrólysis" (cuadrado vacío) es relativamente inefectiva para fijar $\mathrm{Na}$, aunque la razón de ello no esté clara. Las escorias y la puzolaza natural son neutras, aunque las dos PFA utilizadas - una baja y la otra alta en $\mathrm{Na}$ - dan sodio fijado en proporción considerable. Aún cuando los PFA han reaccionado incompletamente a los 14 meses, se puede predecir que la capacidad de retención de los álcalis por esas adiciones, mejorará con la edad. La referencia (9) también da información sobre el $K$, que se comporta algo diferente al $\mathrm{Na}$; se están obteniendo datos para las mismas adiciones, pero con un cemento alto en álcalis.

\section{DURABILIDAD DE LOS CEMENTOS $Y$ CEMENTOS CON ADICIONES ACTIVAS}

Las investigaciones sobre cementos efectuadas en la pasada década han demostrado en forma creciente la importancia de los cambios químicos en la durabilidad del should fall on the dashed line. However, OPC lies much below this line, indicating that much of the $\mathrm{Na}$ is retained by the solid hydration products. Analytical electron microscopy discloses that most of the $\mathrm{Na}$ is in the $\mathrm{C}-\mathrm{S}-\mathrm{H}$ phase, where it presumably replaces $\mathrm{Ca}$. The effect of $15 \%$ modifier is also shown. Silica fume (solid circle) not only contains little $\mathrm{Na}$, but substantially increase the sorption potential of the system for $\mathrm{Na}$. Flame hydrolysis silica (open square) is relatively ineffective in remvoing $\mathrm{Na}$, although the reasons for this are far from clear. Slag and natural pozzolan are broadly neutral, but the two PFA's used -one low in $\mathrm{Na}$, the other high- both give substantial sodium removal. Although the PFA's are still incompletely reacted at 14 months, it is predicted that the alkali retention power of these blends will continue to improve with age. The reference (9) also shows data for $K$, which behaves somewhat differently than $\mathrm{Na}$; data for the same blends, but made with a high alkali cement, are being obtained.

\section{DURABILITY OF CEMENTS AND BLENDED CEMENTS}

Research on cements during the past decade has shown increasingly the importance of chemical changes in the durability of cement and concrete. The potential lowering of the 
cemento y del hormigón. Un ejemplo es la disminución del $\mathrm{pH}$ interno de los cementos a causa de la actividad de adiciones silícicas, así como la del potencial redox que intínsecamente existe en los cementos. Las reacciones ambientales suponen con frecuencia cambios químicos. Otro ejemplo es la pérdida gradual de los álcalis que tienen los líquidos de los poros por lixiviación, así como la disolución del $\mathrm{Ca}(\mathrm{OH})_{2}$, fenómenos ambos que hacen disminuir el pH interno.

Otros cambios habituales -carbonatación, fijación de cloruros y sulfatos- suponen una mejor reconstrucción de textura y mineralógica de las fases sólidas que componen la matriz cementicia. Las reacciones secundarias deben tener una importancia considerable; por ejemplo los áridos susceptibles de reaccionar con los álcalis, sufren expansión en contacto con cementos, especialmente si éstos tienen un elevado contenido de álcalis. A causa de la importancia de este fenómeno se han desarrollado diversos simposios internacionales, el más reciente en 1984, que han analizado las causas, y su control y protección. internal $\mathrm{pH}$ of cements brought about by the addition of siliceous blending agents is one example, as is the redox potential which exists within cements. Environmental reactions, frequently involve chemical changes. Examples include the gradual loss by leaching of alkali from pore fluids and solution of $\mathrm{Ca}(\mathrm{OH})_{2}$, all of which lower the internal $\mathrm{pH}$. The other common environmental changes,

-carbonation, uptake of chloride and sulphate- all involve major textural and mineralogical reconstitutions of the solid phases which comprise the cementing matrix. Secondary reactions may be of considerable importance; some alkali-susceptible aggregates, for example, deteriorate with swelling in contact with cement, especially high-alkali cements. On account of the importance of the phenomena, several international symposia, themost recent in 1984, have been held to analyze its causes, prevention and control.

At the same time, durability has important physical aspects. The rate of penetration into concrete of, for example, chloride ions from sea water, is dependent in part on the permeability of the concrete. Blending agents can play an important role in reducing the

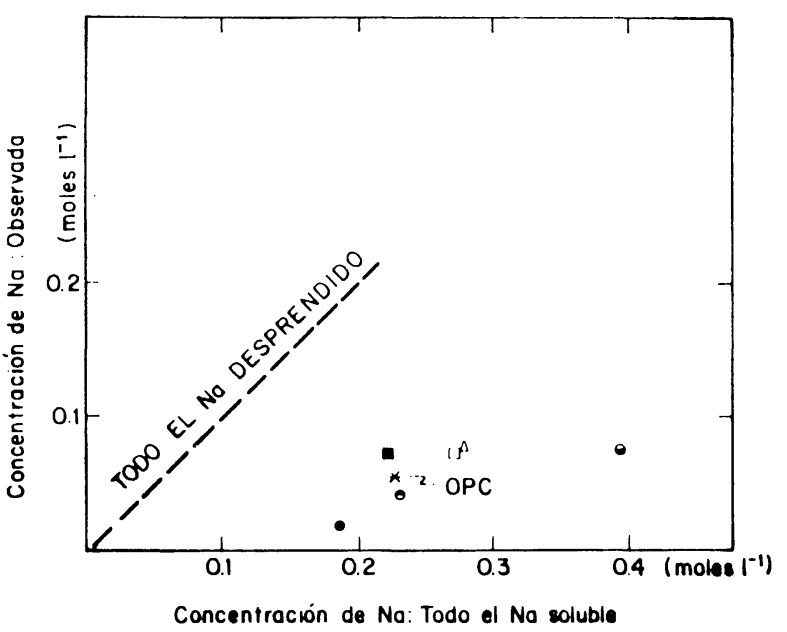

\footnotetext{
- humo de sílice.

escoria de alto horno.

"flame hidrolisis sílica"

$\triangle$ puzolana natural.

ceniza volante baja en álcalis

ceniza volante alta en álcalis.
}

Fig. 2.-Distribución de $\mathrm{Na}$ entre los productos de hidratación sólidos y la fase acuosa de un OPC bajo en $\mathrm{Na}$ y de cementos con adiciones activas, después de 14 meses de envejecimiento. Las adiciones activas, añadidas al cemento en un $15 \%$ en peso, son:

Al mismo tiempo, la durabilidad tiene importantes aspectos físicos. Por ejemplo, la velocidad de penetración dentro del hormigón de los iones $\mathrm{Cl}^{-}$del agua de mar, que

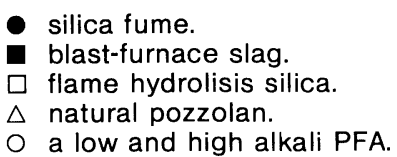

Fig. 2.-The distribution of $\mathrm{Na}$ between solid hydration products and aqueous phase of a low-Na OPC and blended cements after 14 months ageing. The blending agents, added at a constant $15 \%$ by weight of cement, are.

permeability of concrete, but will not automatically do so unless rules for their formulation, mixing, emplacement and curing are correctly formulated and rigidly adhered to 
depende parcialmente de la permeabilidad de aquel hormigón. Las adiciones activas pueden jugar un importante papel reduciendo la permeabilidad, aunque no sistemáticamente, a menos que las normas para su dosificación, mezcla, emplazamiento y curado se hagan correctamente, y se eleven a la práctica con rigidez. Hasta el día de hoy, gran parte de la experiencia adquirida con estos materiales es semi-empírica, pero el conocimiento debe de plantearse en muchos casos, desde un punto de vista científico. Las interacciones físicas y químicas de los cementos suponen un fértil campo de futuras investigaciones. in practice. At present, much of the experience gained with these materials is semi-empirical, but eventually it must be placed on a proper scientific footing. The interactions between chemical and physical actions in cement remains a fertile field for further research.

\section{REFERENCIAS}

(1) R. M. HERATH BANDA and F. P. GLASER: Cement and Concrete Res. 8 319-324, 1978

(2) R. KONDO: et al. American Ceramic Society Symposium, Chicago (1976).

(3) R. PAZDEJ and F. P. CORRENTINO: IRSID Report, Contract EEB 4 (1981)

(4) E. BRINER and N. GAGNEUX: Helv. Chem. Acta 31 (2), 556 (1968).

(5) F. P. GLASSER and E. SHAME: Brit. Patent 8409356 (April 11, 1984)

(6) E. SHAME and F. P. GLASSER: Advances in Cement Res. (in press).

(7) M. PEREZ MENDEZ. R. A. HOWIE and F. P. GLASSER: Cement Concr. Res.. 14 57-63 (1984).

(8) J. FAYOS. F. P. GLASSER. R. A. HOWIE. E. LACHOWSKI and M. PEREZ MENDEZ: Acta Crystallog. B (in press).

(9) F. P. GLASER and J. MARR: pp. 419-429 in "Chemistry and chemically Related Properties of Cement" Proceedings Brit. Ceram. Soc.. Vol. 35 (1984)

(10) F. P. GLASSER and J. MARR: pp. 239-42 in "Alkalis in Concrete" (Proc. 6th Intl. Symp. on Alkali-Aggregate Reactions) Copenhagen, DBF. (1983).

\section{publicación del I.E.T.c.c.}

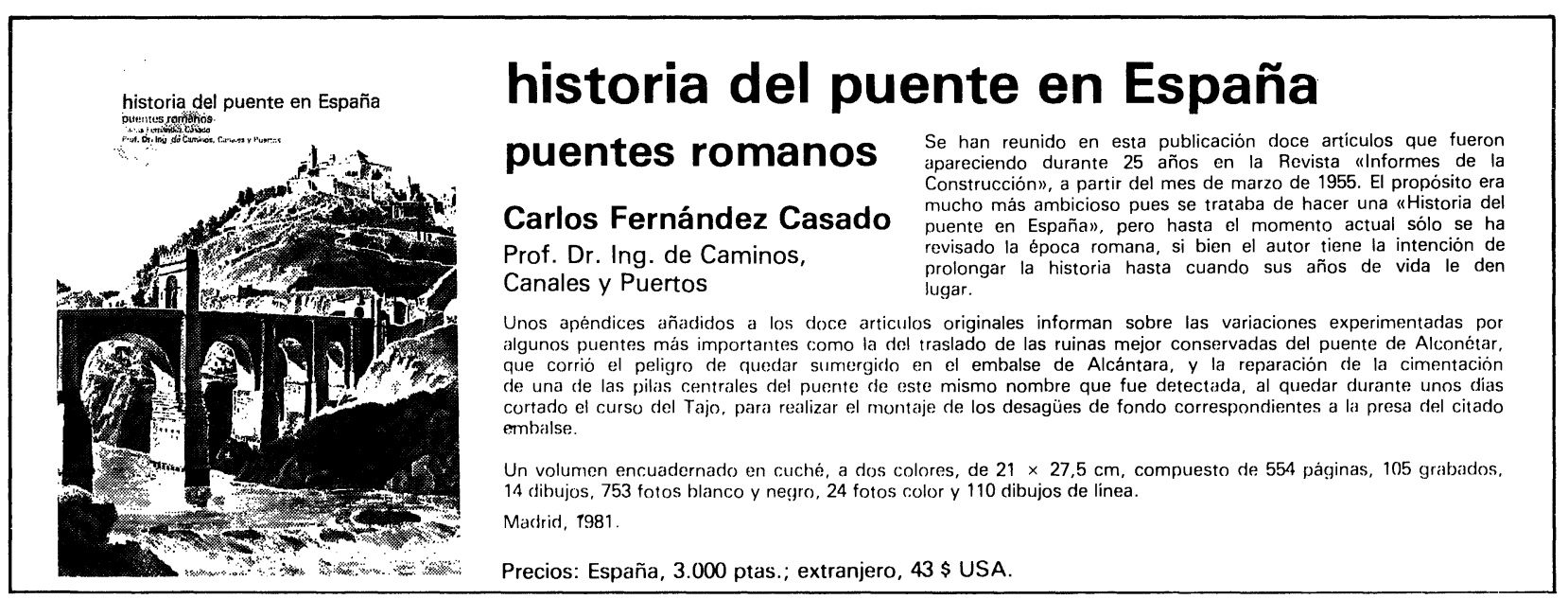

